\author{
Yoshito OHNO ${ }^{1)}$, Yuichiro AKUNE ${ }^{1)}$, Hiroshi NITTO') and Yasuo INOSHIMA ${ }^{2-4) *}$ \\ 1)Port of Nagoya Public Aquarium, 1-3 Minato-machi, Minato-ku, Nagoya, Aichi 455-0033, Japan \\ ${ }^{2)}$ Cooperative Department of Veterinary Medicine, Gifu University, 1-1 Yanagido, Gifu 501-1193, Japan \\ 3)The United Graduate School of Veterinary Sciences, Gifu University, 1-1 Yanagido, Gifu 501-1193, Japan \\ 4)Education and Research Center for Food Animal Health, Gifu University (GeFAH), 1-1 Yanagido, Gifu 501-1193, \\ Japan
}

J. Vet. Med. Sci.

81(3): 449-453, 2019

doi: 10.1292/jvms.18-0391

Received: 9 July 2018

Accepted: 7 January 2019

Published online in J-STAGE:

22 January 2019
ABSTRACT. A bottlenose dolphin (Tursiops truncatus) housed in the Port of Nagoya Public Aquarium (PNPA) presented with symptomatic pneumonia caused by Aspergillus fumigatus. The dolphin was treated with micafungin. On days 2 and 11 after the first administration of micafungin, results from a physical examination and laboratory test indicated a decline of body temperature (BT) and leukopenia, with lowest BT, white blood cells (WBCs), and segmented neutrophils (SEGs) of $34.2^{\circ} \mathrm{C}, 600$ cells $/ \mu l$, and 67 cells $/ \mu l$, respectively. BT, WBCs, and SEGs returned to normal range after administration of granulocyte colony stimulating factor (G-CSF). To the best of our knowledge, this is the first report of micafungin-induced decline of BT and leukopenia that was successfully treated with G-CSF in a bottlenose dolphin.

KEY WORDS: bottlenose dolphin, fungal pneumonia, G-CSF, leukopenia, micafungin

Fungal pneumonia is a common disease in bottlenose dolphins (Tursiops truncatus), which is most commonly caused by Aspergillus fumigatus [15]. Various antifungals, such as flucytosine, itraconazole, voriconazole, and posaconazole are used for the treatment of respiratory fungal infection in cetaceans $[2,12]$. In clinical treatment of cetaceans, hematological abnormalities (leukopenia, neutropenia, and thrombocytopenia) have been reported with some drugs including antifungal agents [6, 10]. Moreover, there are some reports about use of granulocyte colony stimulating factor (G-CSF) for the treatment of these side effects in cetaceans $[10,11]$.

Micafungin is used for the treatment of some types of fungal infections including A. fumigatus in humans. Some studies have indicated that micafungin has a high clinical efficacy and safety in the treatment of aspergillosis in humans [1, 8]; however, based on the European Medicines Agency report, associated side effects include elevated hepatic enzyme(s) levels and leukopenia [4]. The use of micafungin treatment and its side effects in bottlenose dolphins has not been previously reported, although it is used with the same dosage ( $3 \mathrm{mg} / \mathrm{kg} / \mathrm{day})$ in this species without side effects, including hematological abnormalities, in other facilities in Japan (personal communication). In this report, we describe our experience with a case of micafungin-induced decline of body temperature (BT) and leukopenia in an A. fumigatus-infected bottlenose dolphin, which showed a good response to G-CSF therapy.

An adult female bottlenose dolphin, estimated to be 13 to 16 years old and weighing $240 \mathrm{~kg}$, was housed in the Port of Nagoya Public Aquarium (PNPA). On day 1 of the disease course, the animal showed lethargy, sputum discharge, and bad breath. Subsequently, a blood test was performed, even though the animal's BT was in the normal range. The animal showed non-specific signs of inflammation, including elevation of white blood cells (WBCs), segmented neutrophils (SEGs), and fibrinogen (FIB) and subsequently developed a fever (Fig. 1). Since A. fumigatus was isolated from the blowhole, as described below, voriconazole (5 $\mathrm{mg} / \mathrm{kg}$ /day, orally; VFEND, Pfizer Japan, Tokyo, Japan) and micafungin (3 mg/kg/day, intravenously; Fungurd, Astellas Pharma, Tokyo, Japan) were administered to the animal on days 2-5, 27-29, 41, and 49 and on days 11-20, respectively. This therapy was combined with amphotericin B (0.2-0.4 mg/kg/day, inhalation; FUNGIZON, Bristol-Myers Squibb, Tokyo, Japan) on days 7-37 (Figs. 1 and 2) using an inhaler device (INH001, Taylor's Pharmacy, Orlando, FL, U.S.A.) made specifically for dolphins [3]. After administration of micafungin, decline of BT and leukopenia was observed. Therefore, the animal was treated with G-CSF (2.5 $\mu \mathrm{g} /$ kg/day, intramuscular; Filgrastim, Mochida Pharmaceutical, Tokyo, Japan) on days 23-25 (first therapy) and days 31-34 (second therapy) for the purpose of stimulating blood cell production in the bone marrow (Figs. 1 and 2).

BT was measured rectally using a digital clinical thermometer with a probe, as described previously [7]. Blood samples were

*Correspondence to: Inoshima, Y.: inoshima@gifu-u.ac.jp

O2019 The Japanese Society of Veterinary Science

This is an open-access article distributed under the terms of the Creative Commons Attribution Non-Commercial No Derivatives (by-nc-nd) License. (CC-BY-NC-ND 4.0: https://creativecommons.org/licenses/by-nc-nd/4.o/) 

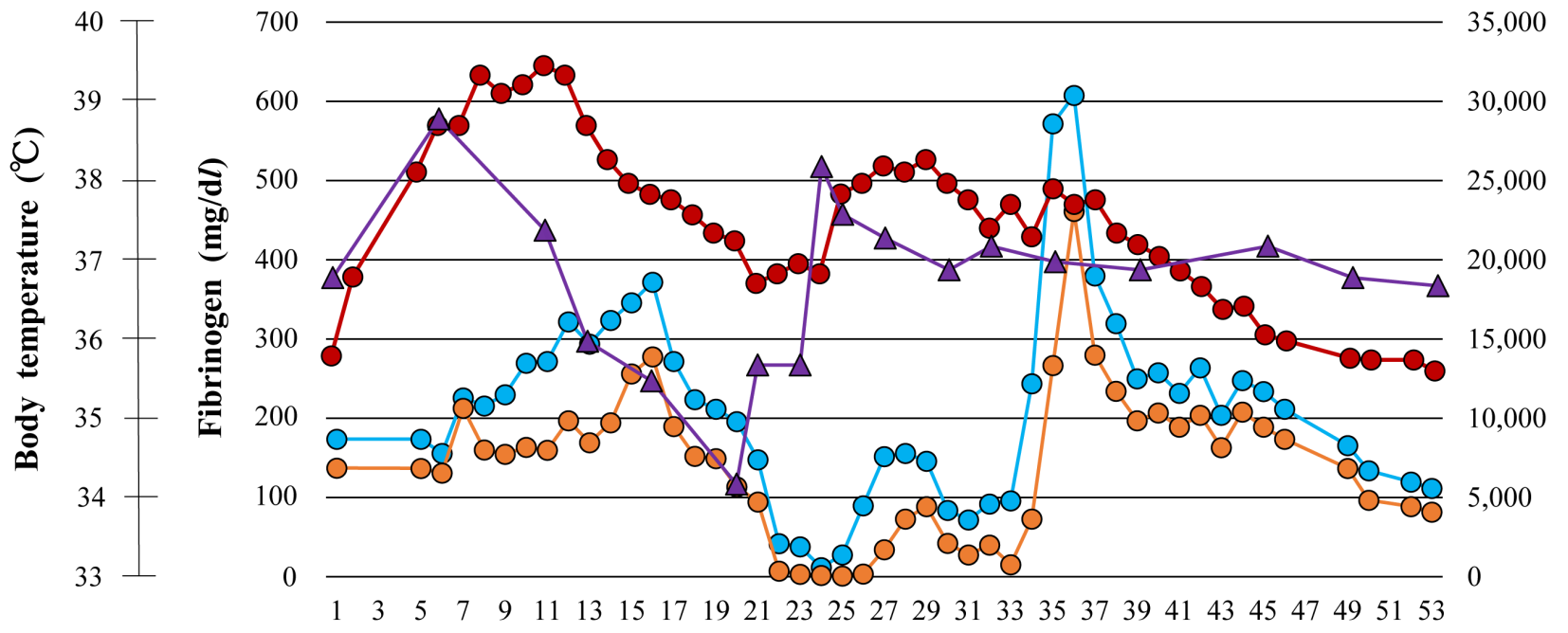

35,000
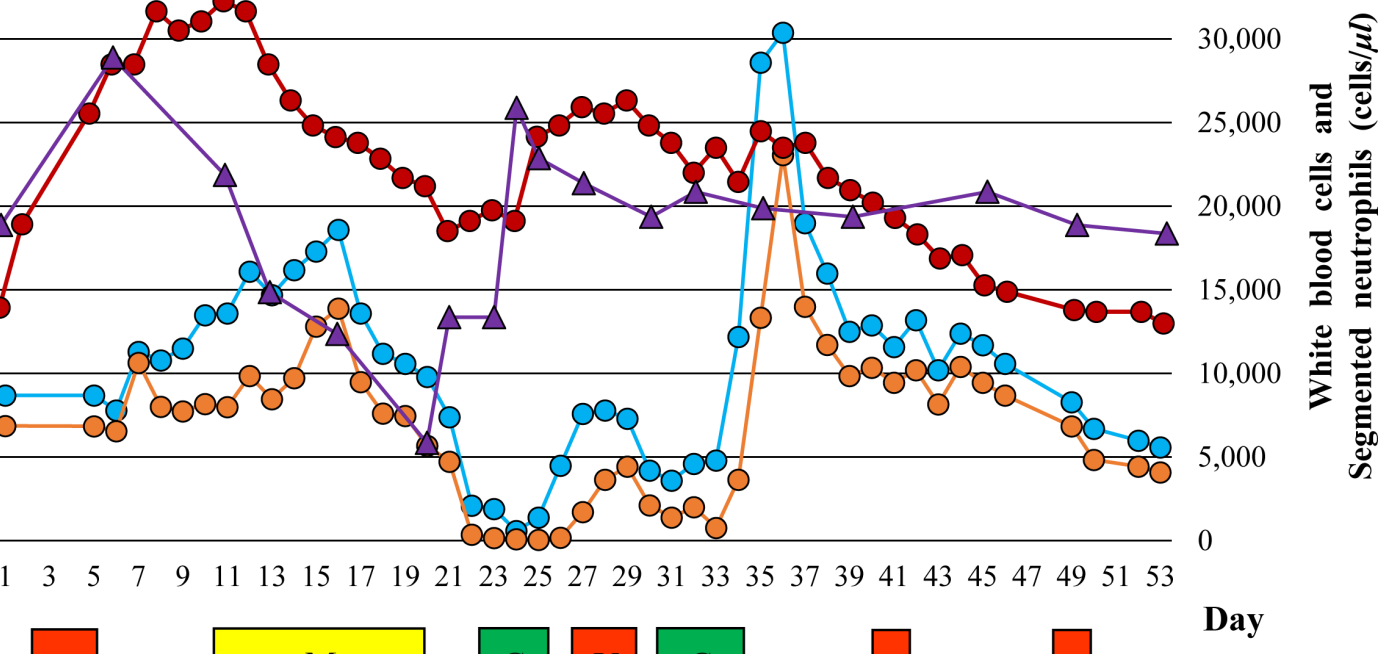

Medications
Antifungals, G-CSF
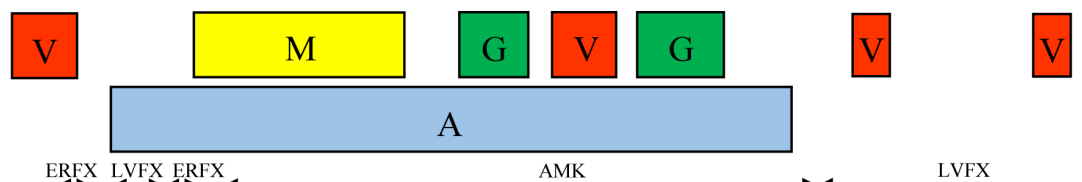

Day

Antibiotics

\section{Side effects}

Clinical symptoms

Lethargy

Sputum

Bad breath

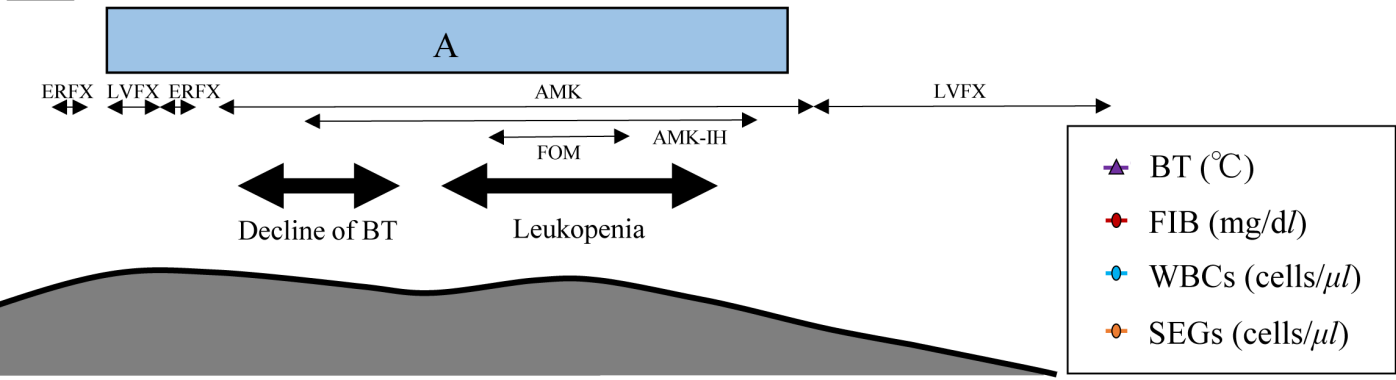

Clinical examinations

Blowhole culture

(A. fumigatus)

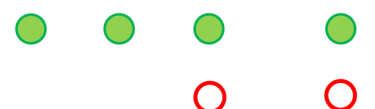

Blood culture

Fig. 1. Clinical change in body temperature (BT) $\left({ }^{\circ} \mathrm{C}\right)$, white blood cells (WBCs) (cells/ $\left.\mu l\right)$, segmented neutrophils (SEGs) $($ cells $/ \mu l)$ and fibrinogen (FIB) $(\mathrm{mg} / \mathrm{d} l)$ during medications with side effects, clinical symptoms, and the results of clinical examinations in a bottlenose dolphin (Tursiops truncatus). The red squares with a $\mathrm{V}$, the yellow square with an $\mathrm{M}$, the blue square with an $\mathrm{A}$, and the green squares with a $\mathrm{G}$ indicate periods of treatment with voriconazole ( $5 \mathrm{mg} / \mathrm{kg} / \mathrm{day}$, orally), micafungin ( $3 \mathrm{mg} / \mathrm{kg} /$ day, intravenously), amphotericin B $(0.2-0.4 \mathrm{mg} / \mathrm{kg} / \mathrm{day}$, inhalation), and granulocyte colony stimulating factor (G-CSF) $(2.5 \mu \mathrm{g} / \mathrm{kg} /$ day, intramuscular) administration, respectively. The thin black arrows indicate antibiotic administration (ERFX: enrofloxacin, LVFX: levofloxacin, AMK: amikacin, AMK-IH: amikacin-inhalation, FOM: fosfomycin). The thick black arrows indicate side effects (decline of body temperature (BT), leukopenia). The gray wave profile indicates the withdrawal of clinical symptoms (lethargy, sputum, bad breath). This profile was based on the subjective assessment data (medical or training records, memories of a veterinarian and dolphin trainers). The green solid and outlined circles indicate the time points at which blowhole fungal cultures were positive or negative for Aspergillus fumigatus, respectively. The red outlined circles indicate the blood cultures are negative.

obtained from the fluke vessels through a 21 -gauge butterfly needle and $10-\mathrm{m} l$ disposable syringe. The blood was collected in ethylene diamine tetra-acetic acid (EDTA) - $2 \mathrm{~K}$ tubes, sodium heparin-coated tubes, $3.2 \%$ sodium citrate tubes, and blood culture bottles (BACT/ALERT FA PLUS, BACT/ALERT FN PLUS, bioMérieux Japan, Tokyo, Japan) for performing a complete blood count, serum biochemistry or measurement of plasma concentration of voriconazole, measurement of FIB, and blood culture, respectively. Blood smears were stained with May-Gruenwald's and Giemsa's stain solution for obtaining the differential WBCs count. Blowhole swab samples on days $1,5,9,15,23,37,45$, and 53 were cultured on Sabouraud agar plates at $37^{\circ} \mathrm{C}$ for $48 \mathrm{hr}$. All examinations except measurement of plasma concentration of voriconazole and blood culture were carried out at the PNPA. The plasma concentrations of voriconazole on days $6,13,21,30,37,41,42,49,50$, and 53 were measured by a clinical testing company (SRL, Tokyo, Japan) through high performance liquid chromatography (HPLC) (HPLC SYSTEM, 10Ave series, Shimadzu, Hamamatsu, Japan). The blood cultures on days 9, 15, 23, 26, 35, and 45 were conducted with BACT/ALERT 3D (bioMérieux Japan, Tokyo, Japan) by a clinical testing company (Health Sciences Research Institute, Yokohama, Japan). Bacterial species identification and drug sensitivity tests on days $1,5,9,15,23,37,45$, and 53 were also conducted by the same company, together with the blood cultures.

A. fumigatus was isolated on days 1, 5, 9, 15, 23, and 37 (Fig. 1). The fungal species was identified based on colony morphology 
Table 1. Summary of administered antibiotics and identity of bacteria isolated from blowhole swab samples

\begin{tabular}{|c|c|c|c|c|}
\hline Day & Antibiotic $^{a)}$ & Dosage $(\mathrm{mg} / \mathrm{kg})$ & Frequency and route ${ }^{\mathrm{c})}$ & Bacterial isolation $^{\mathrm{e})}$ \\
\hline 5,10 & Enrofloxacin & 5.0 & SID, IM & V. fluvialis, S. intermedius (1) \\
\hline $7-9,39-52$ & Levofloxacin & 7.0 & SID, PO & $\begin{array}{l}\text { V. fluvialis, } \alpha \text {-Streptococcus }(5) \\
\text { A. faecalis, } \alpha \text {-Streptococcus }(9)\end{array}$ \\
\hline $12-38$ & Amikacin & 11.0 & SID, IM & A. caviae, $P$. aeruginosa $(15,23)$ \\
\hline $16-36$ & Amikacin $^{\text {b) }}$ & $0.8-0.9$ & $\mathrm{SID}, \mathrm{IH}^{\mathrm{d})}$ & $\begin{array}{l}\alpha \text {-Streptococcus, } P \text {. aeruginosa }(37) \\
\text { A. caviae, } P \text {. aeruginosa }(45)\end{array}$ \\
\hline $24-30$ & Fosfomycin & 20.0 & BID, IV & Corynebacterium $s p, P$. aeruginosa (53) \\
\hline
\end{tabular}

a) Enrofloxacin: Baytril one-shot, Bayer Yakuhin, Osaka, Japan; Levofloxacin: CRAVIT, DAIICHI SANKYO COMPANY, Tokyo, Japan; Amikacin: AMIKACIN SULFATE, Fuji Pharma, Tokyo, Japan; Fosfomycin: FOSMICIN, Meiji Seika Pharma, Tokyo, Japan; b) 4-fold diluted amikacin with saline was used; c) SID: once a day; BID: twice a day; IM: intramuscular administration; PO: oral administration; IH: inhalation; IV: intravenous administration; d) inhaler device made specifically for dolphins [3] was used; e) Number in parenthesis indicates the day of bacterial isolation; V. fluvialis: Vibrio fluvialis; S. intermedius: Staphylococcus intermedius; A. faecalis: Alcaligenes faecalis; A. caviae: Aeromonas caviae; P. aeruginosa: Pseudomonas aeruginosa.

[16] at the PNPA. Some bacteria including Pseudomonas aeruginosa were isolated from the blowhole swab samples, and antibiotics were selected according to the results of the drug sensitivity tests (Table 1). The blood cultures were all negative (Fig. $1)$.

The highest BT of the dolphin $\left(38.8^{\circ} \mathrm{C}\right)$ was recorded on day 6 . A decline of BT $\left(36.0^{\circ} \mathrm{C}\right)$ was initially recorded on day 13 , with lowest BT $\left(34.2^{\circ} \mathrm{C}\right)$ on day 20 , two and nine days after the first administration of micafungin, respectively. After stopping micafungin administration, there was no further decline of BT; subsequently, the BT increased to $35.7^{\circ} \mathrm{C}$ on day 21 . Although the BT showed a transient increase to $38.2^{\circ} \mathrm{C}$ on day 24 during the first G-CSF therapy, it decreased gradually to $36.7^{\circ} \mathrm{C}$ by day 53 , which was within normal range for this animal (Fig. 1).

A decrease in WBCs and SEGs was observed on day 22, eleven days after the first administration of micafungin, with lowest values of 600 cells $/ \mu l$ and 67 cells/ $\mu l$ on days 24 and 25, respectively. Six days after the first administration of G-CSF (day 29), WBCs and SEGs increased to 7,300 cells/ $\mu l$ and 4,446 cells/ $\mu l$, respectively, which were within normal ranges for this animal; however, since both values showed a subsequent decline to 3,600 cells $/ \mu l$ and 773 cells $/ \mu l$ on days 31 and 33 , respectively, the second G-CSF therapy was conducted (days 31-34). Thereafter, WBCs and SEGs showed a transient increase to 30,400 cells/ $\mu l$ and 23,074 cells $/ \mu l$ on day 36 and decreased gradually by day 50 to 6,700 cells $/ \mu l$ and 4,844 cells $/ \mu l$, respectively, which were within normal ranges for this animal (Fig. 1). After starting micafungin administration, a hepatic enzyme, glutamic oxaloacetic transaminase (GOT) increased significantly and attained maximum level (412 IU/l) on day 21 (Fig. 2). The plasma concentrations of voriconazole on days $6,13,21,30,37,41,42,49,50$, and 53 were $8.55,5.09,2.30,12.94,5.68,3.36,6.99,2.79,6.16$, and 4.30 $\mu \mathrm{g} / \mathrm{ml}$, respectively (Fig. 2).

Various antifungals are used for the treatment of respiratory fungal infection in cetaceans $[2,12]$. To our knowledge, there are no previous reports of the use of micafungin for the treatment of fungal infection in cetaceans. In this report, micafungin was selected for treatment because there were no improvements in the BT, blood test results (WBCs, SEGs, and FIB), clinical symptoms, and blowhole fungal culture after voriconazole administration (Fig. 1), a commonly used treatment for fungal infection in cetaceans [2, 14]. Decline of BT and leukopenia were only observed after micafungin administration (Figs. 1 and 2). These phenomena might be caused by micafungin but not by voriconazole because they were not observed immediately post voriconazole administration: plasma concentration of voriconazole $(8.55 \mu \mathrm{g} / \mathrm{m} l)$ (Fig. 2) was above the safe range (2 to $4 \mu \mathrm{g} / \mathrm{m} l)$ in this species [5], although the cause or action mechanism of these side effects remains unknown. The first decline of BT was observed on day 13, when the plasma concentration of voriconazole was $5.09 \mu \mathrm{g} / \mathrm{ml}$ (Fig. 2), which exceeded both the safe range and upper limit (5 $\mu \mathrm{g} / \mathrm{m} l)$, increasing the risk of hepatic side effects in this species [5]. However, we observed no other side effects including anorexia or visual abnormalities which are the main known adverse effects of voriconazole $[5,13]$. Moreover, there was no further decline of BT after withdrawing micafungin (Figs. 1 and 2). With regard to the GOT levels, a maximum level was observed on day 21, just after micafungin administration, rather than after voriconazole administration (Fig. 2). Ferrier et al. [5] reported that GOT level showed a possible relationship with voriconazole concentration in bottlenose dolphins; however, at the time of the assay, the plasma concentration of voriconazole was within the safe range $(2.30 \mu \mathrm{g} / \mathrm{ml})$ (Fig. 2). In our study, the plasma concentration of voriconazole (maximum level, $12.94 \mu \mathrm{g} / \mathrm{m} l$ ) (Fig. 2) showed a transient increase above the safe range during the treatment period. However, a previous report had indicated that a plasma concentration of voriconazole of 9 to $12 \mu \mathrm{g} / \mathrm{m} l \mathrm{was}$ tolerable for this species [13]. Furthermore, there were no other side effects including anorexia, visual abnormalities, and further elevation of the GOT levels during the treatment (Fig. 2). Focusing on the differential WBCs count in this case, eosinophilia and lymphocytosis were also seen prominently with highest values of 4,101 cells/ $\mu l$ and 2,365 cells $/ \mu l$ on days 13 and 14 , respectively, during micafungin administration (data not shown). These phenomena might be caused by a hypersensitive reaction to micafungin. Similar reactions to sulfonamide have been reported in killer whale (Orcinus orca), dogs, and humans with neutropenia and thrombocytopenia [10], as well as to vancomycin in humans with neutropenia and eosinophilia [9]. Furthermore, the values of both the eosinophils and of the lymphocytes decreased to normal ranges for this animal after withdrawing micafungin. It is likely that the observed decline of BT and leukopenia in this animal was related to the side effects of micafungin rather than to those of voriconazole and other administered drugs. 

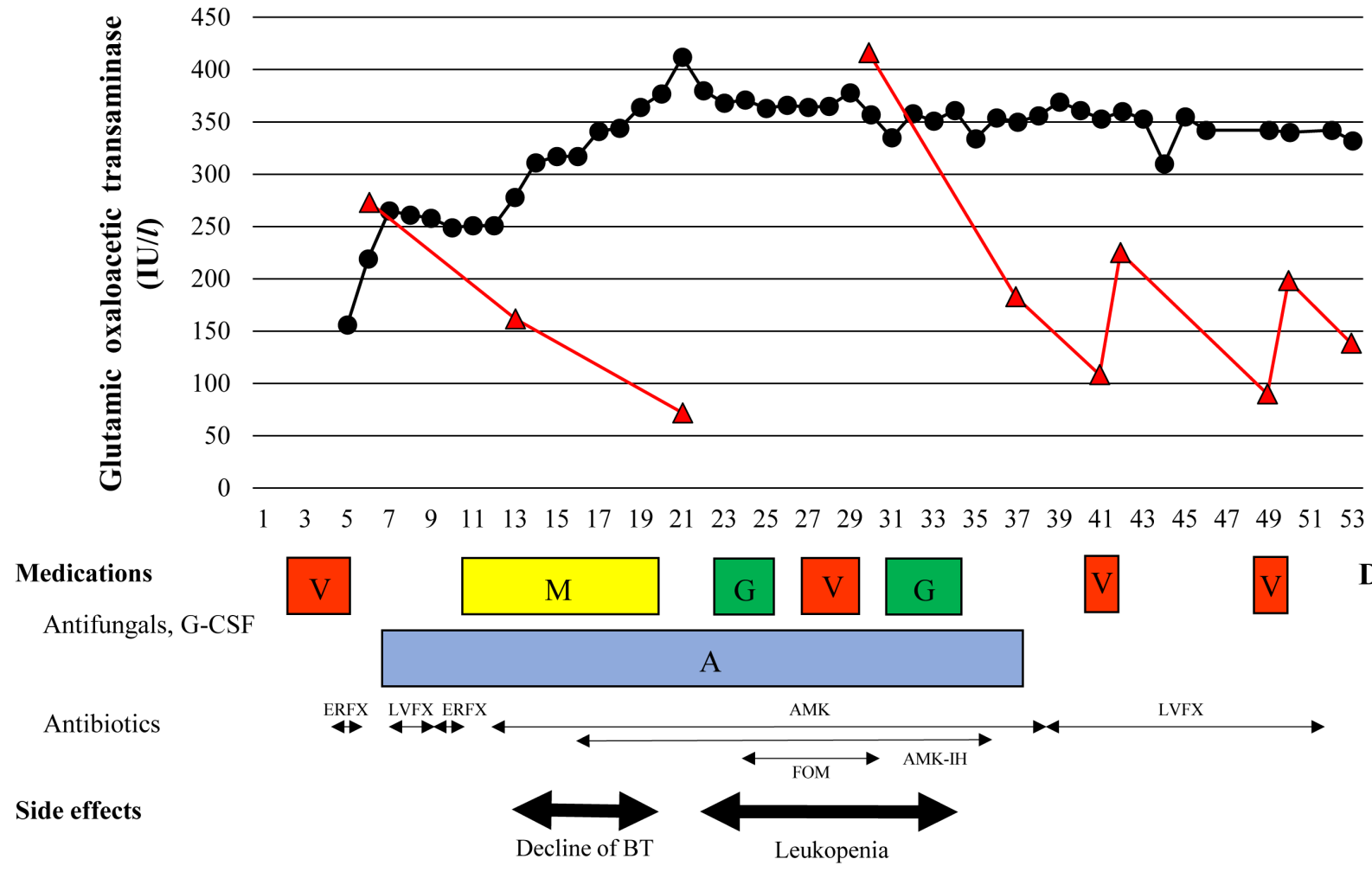

Day

$$
\begin{aligned}
& \text { - GOT (IU/l) } \\
& \text { * Voriconazole concentration }(\mu \mathrm{g} / \mathrm{m} l)
\end{aligned}
$$

Fig. 2. Change in glutamic oxaloacetic transaminase (GOT) levels (IU/l) and voriconazole concentrations $(\mu \mathrm{g} / \mathrm{m} l)$ in blood during drug therapy with side effects in a bottlenose dolphin (Tursiops truncatus). The red squares with a $\mathrm{V}$, the yellow square with an $\mathrm{M}$, the blue square with an $\mathrm{A}$, and the green squares with a $\mathrm{G}$ indicate periods of treatment with voriconazole ( $5 \mathrm{mg} / \mathrm{kg} /$ day, orally), micafungin ( $3 \mathrm{mg} / \mathrm{kg} / \mathrm{day}$, intravenously), amphotericin B (0.2-0.4 mg/kg/day, inhalation), and granulocyte colony stimulating factor (G-CSF) (2.5 $\mu \mathrm{g} / \mathrm{kg} /$ day, intramuscular) administration, respectively. The thin black arrows indicate antibiotic administration (ERFX: enrofloxacin, LVFX: levofloxacin, AMK: amikacin, AMKIH: amikacin-inhalation, FOM: fosfomycin). The thick black arrows indicate side effects (decline of body temperature (BT), leukopenia).

Ultimately, this animal recovered from its A. fumigatus infection owing to a combination therapy with voriconazole (systemic) and amphotericin B (topical). Treatment success was judged from normalization of the BT, blood test results (WBCs, SEGs, and FIB), and clinical symptoms and the disappearance of $A$. fumigatus from the blowhole (Fig. 1). It was very difficult to evaluate the efficacy of micafungin because it was discontinued post appearance of the side effects. However, we can say for sure that it is very important to monitor the BT and the hematological panels regularly during micafungin administration. Moreover, the effectivity of G-CSF for treating drug-induced leukopenia in cetaceans, as described previously [10], was reaffirmed through this case.

In conclusion, we present the first report of a micafungin-induced decline of BT and leukopenia in a bottlenose dolphin.

ACKNOWLEDGMENTS. We would like to acknowledge Dr. Todd L. Schmitt (Sea World San Diego, San Diego, CA, U.S.A.) for providing his expertise. We wish to thank all the members of the dolphin team of the Port of Nagoya Public Aquarium. This study was partly supported by the Grant for Joint Research Program of the Research Center for Zoonosis Control, Hokkaido University, from the Ministry of Education, Culture, Sports, Science and Technology, Japan.

\section{REFERENCES}

1. Aikawa, N., Kusachi, S., Oda, S., Takesue, Y. and Tanaka, H. 2008. Clinical effects of micafungin, a novel echinocandin antifungal agent, on systemic fungal infections in surgery, emergency, and intensive-care medicine; Evaluation using the AKOTT algorithm. Jpn. J. Chemother. 56: 330-343.

2. Bunskoek, P. E., Seyedmousavi, S., Gans, S. J. M., van Vierzen, P. B. J., Melchers, W. J. G., van Elk, C. E., Mouton, J. W. and Verweij, P. E. 2017. Successful treatment of azole-resistant invasive aspergillosis in a bottlenose dolphin with high-dose posaconazole. Med. Mycol. Case Rep. 16: 16-19. [Medline] [CrossRef] 
3. Dold, C., Deaver, A., Gearhart, S., Croft, L. and McBain, J. 2008. Development and use of a "pool-side" nebulized drug delivery system for bottlenose dolphins (Tursiops truncatus). pp. 160-161. In: 39th Annual International Association for Aquatic Animal Medicine Conference Proceedings, Pomezia.

4. European Medicines Agency. 2011. Mycamine: EPAR summary for the public. http://www.ema.europa.eu/docs/en_GB/document_library/ EPAR_-_Summary_for_the_public/human/000734/WC500031077.pdf [accessed on February 15, 2018].

5. Ferrier, K. R. M., van Elk, C. E., Bunskoek, P. E. and van den Broek, M. P. H. 2017. Dosing and therapeutic drug monitoring of voriconazole in bottlenose dolphins (Tursiops truncatus). Med. Mycol. 55: 155-163. [Medline] [CrossRef]

6. Fothergill, M. and Jogessar, V. B. 1986. Haematological changes in two Lagenorhynchus obscurus treated with ketoconazole. Aquat. Mamm. 12: $87-91$.

7. Katsumata, E. 2010. Study on reproduction of captive marine mammals. J. Reprod. Dev. 56: 1-8. [Medline] [CrossRef]

8. Kohno, S., Niki, Y., Amitani, R., Ogawa, K., Kurashima, A. and Miyazaki, Y. 2010. Clinical efficacy and safety of micafungin, a novel echinocandin antifungal drug, in pulmonary aspergillosis in a postmarketing setting. Jpn. J. Chemother. 58: 128-139.

9. Lai, K. K., Kleinjan, J. and Belliveau, P. 1996. Vancomycin-induced neutropenia treated with granulocyte colony-stimulating factor during home intravenous infusion therapy. Clin. Infect. Dis. 23: 844-845. [Medline] [CrossRef]

10. Lavergne, S., Reidarson, T. H., Schmitt, T. and McBain, J. 2006. Anti-drug and anti-platelet antibodies in a killer whale with signs of sulfonamide hypersensitivity. pp. 9-10. In: 37th Annual International Association for Aquatic Animal Medicine Conference Proceedings, Nassau.

11. Martelli, P. R. and Hui, S. W. 2018. Update on melioidosis in zoo and wild animals. pp. 315-321. In: Fowler's Zoo and Wild Animal Medicine Current Therapy, Volume 9, 1st ed. (Miller, R. E., Lamberski, N. and Calle, P. eds.), Elsevier, Amsterdam.

12. Reidarson, T. H., McBain, J. F., Dalton, L. M. and Rinaldi, M. G. 2001. Mycotic diseases. pp. 337-355. In: CRC Handbook of Marine Mammal Medicine, 2nd ed. (Dierauf, L. A. and Gulland, F. M. D. eds.), CRC Press, Boca Raton.

13. van Elk, C. E. 2007. Voriconazole dosage in bottlenose dolphins. p. 245. In: 38th Annual International Association for Aquatic Animal Medicine Conference Proceedings, Orlando.

14. van Elk, C. E., Gans, S. J. M. and Epping, N. 2006. A Candida glabrata bronchopneumonia treated with voriconazole in a Tursiops truncatus. pp. 111-112. In: 37th Annual International Association for Aquatic Animal Medicine Conference Proceedings, Nassau.

15. Venn-Watson, S., Daniels, R. and Smith, C. 2012. Thirty year retrospective evaluation of pneumonia in a bottlenose dolphin Tursiops truncatus population. Dis. Aquat. Organ. 99: 237-242. [Medline] [CrossRef]

16. Yaguchi, T. 2011. Aspergillus. Clin. Micobiol. 38: 537-546. 